\title{
Lymphoscintigraphy: Breast and Melanoma
}

Kathy S. Thomas and Jessica Williams

$\mathbf{L}$ (sentinel node) receiving lymphatic drainage from a tumor site and can provide the surgeon with a map to follow during surgery and help stage the disease $(1,2)$.

\section{CLINICAL INDICATIONS}

- Identification of the sentinel lymph node for surgical excision to stage the disease.

- Assistance in planning the incision for sentinel node biopsy.

- Evaluation of intermediate-stage primary melanoma.

\section{CONTRAINDICATIONS}

- Patients who are pregnant or breastfeeding. Pregnancy must be excluded in accordance with local institutional policy. If the patient is breastfeeding, appropriate radiation safety instructions should be provided.

- Patients with known metastases.

- Patients who have recently undergone a radiopharmaceutical-dependent nuclear medicine study.

\section{PATIENT PREPARATION AND EDUCATION}

- Patient may eat and take medications as prescribed $(4,5)$.

- Fasting requirements for same-day surgical procedures should be followed as prescribed by the surgical department.

- A topical numbing agent may be used (lidocaine and prilocaine cream [Emla; AstraZeneca] or cold spray) to minimize pain.

- A focused clinical history should be obtained, including previous biopsy results, the pathologic classification of the tumor (thickness and penetration), and surgical excision, if any, of the primary lesion.

\section{ACQUISITION INSTRUCTIONS}

Breast

- The total dose is divided into 1-4 aliquots (institutionspecific). Adding $\mathrm{pH}$-balanced $1 \%$ lidocaine to the radiopharmaceutical may improve patient comfort without compromising sentinel lymph node identification.

- The injections, performed by the physician or a trained individual, are placed around the tumor or biopsy site approximately $1 \mathrm{~cm}$ from the edge of the lesion and

COPYRIGHT @ 2020 by the Society of Nuclear Medicine and Molecular Imaging. may be subdermal, periareolar, subareolar, or intradermal. Ultrasound guidance may be needed to determine the injection location for nonpalpable lesions.

- Imaging is tracer-dependent and is performed in 2 phases (Tables 1 and 2). Early-phase static or dynamic imaging to identify the first draining node begins immediately after injection of ${ }^{99 \mathrm{~m}} \mathrm{Tc}$-sulfur colloid or $15 \mathrm{~min}$ after

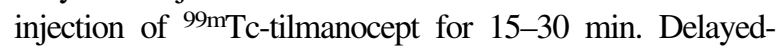
phase imaging to identify lymph node retention and to visualize the sentinel nodes is performed for $30 \mathrm{~min}$ to $3 \mathrm{~h}$, at the conclusion of the initial early-phase imaging sequence for either isotope, in the anterior, oblique, and lateral views.

- Transmission imaging to delineate the patient's body contour using a ${ }^{57} \mathrm{Co}$ sheet source may be helpful for localization. Alternatively, a ${ }^{99 \mathrm{~m}} \mathrm{Tc}$ point source may be used to trace the body contour outline.

- The anterior and lateral locations of the sentinel nodes are marked and labeled on the patient's skin.

- Imaging may not be necessary and is institution-specific (see the "Adjunct Imaging and Interventions" section).

\section{Melanoma}

- The total dose is divided into 4-8 aliquots with a volume of $0.1 \mathrm{~mL}$ each. Each aliquot should contain at least $3,700 \mathrm{kBq}(100 \mu \mathrm{Ci})$ of activity.

- The intradermal injections, performed by the physician or a trained individual, are placed around the tumor or biopsy site approximately $1 \mathrm{~cm}$ from the edge of the lesion.

- Imaging is performed immediately after injection of sulfur colloid or $15 \mathrm{~min}$ after injection of ${ }^{99 \mathrm{~m}} \mathrm{Tc}$-tilmanocept, with the patient supine or prone and with the field of view centered over the region of interest.

- If the injection is on the trunk, all nodal basins are imaged to rule in or rule out lymphatic involvement. If the injection is on a limb, only nodal basins on that limb and the trunk are imaged (e.g., if injecting an ankle, image the popliteal and inguinal nodes).

- A continuous dynamic acquisition (30 s/frame for 30$60 \mathrm{~min}$ ) or sequential static images (every $5 \mathrm{~min}$ for $1 \mathrm{~h}$ ) are obtained until the sentinel lymph node is identified.

- The location of the sentinel node or nodes is marked on the skin.

\section{COMMON OPTIONS}

- Gentle massage at the injection site may be performed to promote tracer uptake (3). 


\begin{tabular}{cll}
\hline Identity & Dose & Administration route \\
\hline 99mTc-sulfur colloid (filtered or nonfiltered) & Mean, $15 \mathrm{MBq}(0.5 \mathrm{mCi})$; range, $15-35 \mathrm{MBq}(0.5-1.0 \mathrm{mCi})$ & Subdermal or intradermal \\
99mTC-tilmanocept & $18.5 \mathrm{MBq}(0.5 \mathrm{mCi})$ & Subdermal or intradermal \\
\hline
\end{tabular}

- The area of injection may need to be covered with a piece of lead to better evaluate the lymphatic drainage.

- Radioactive markers positioned on known anatomic locations may be helpful.

- Oblique and lateral images of the region of interest may be helpful for localization.

TABLE 2

Standard Acquisition Parameters for Dynamic, Static, and Planar Imaging $(1,2)$

\begin{tabular}{|c|c|}
\hline Category & Parameter \\
\hline Camera type & Large-field-of-view multidetector \\
\hline Energy peak & $140 \mathrm{keV}$ \\
\hline Energy window & $20 \%$ \\
\hline Collimator & Low-energy high-resolution \\
\hline \multicolumn{2}{|l|}{ Patient position } \\
\hline Breast & Supine \\
\hline Melanoma & Supine or prone \\
\hline \multicolumn{2}{|l|}{$\begin{array}{c}\text { Camera position, } \\
\text { dual-head }\end{array}$} \\
\hline Detector 1 & Anterior \\
\hline Detector 2 & Posterior \\
\hline $\begin{array}{l}\text { Camera position, } \\
\text { single-head }\end{array}$ & Anterior \\
\hline \multicolumn{2}{|l|}{ Injection-to-scan time } \\
\hline Sulfur colloid & Immediate \\
\hline 99mTc-tilmanocept & 15-min delay \\
\hline Acquisition type & Dynamic or planar \\
\hline \multicolumn{2}{|l|}{ Views } \\
\hline Breast & $\begin{array}{l}\text { Immediate anterior images for } \\
\sim 15-30 \text { min; delayed anterior, } \\
\text { oblique, or lateral images, as } \\
\text { appropriate, for } 3 \mathrm{~h}\end{array}$ \\
\hline Melanoma & $\begin{array}{l}\text { Immediate anterior or posterior } \\
\text { images for } \sim 30-60 \mathrm{~min} \text {; } \\
\text { additional anterior, oblique, or } \\
\text { lateral images, as appropriate, } \\
\text { for } 1 \mathrm{~h}\end{array}$ \\
\hline Matrix & $128 \times 128$ or $256 \times 256$ \\
\hline \multicolumn{2}{|l|}{ Acquisition time } \\
\hline Dynamic: breast & $30 \mathrm{~s} /$ frame for $2-30 \mathrm{~min}$ \\
\hline Dynamic: melanoma & $30 \mathrm{~s} /$ frame for $30-60 \mathrm{~min}$ \\
\hline Planar & $5-10 \mathrm{~min} / \mathrm{view}$ \\
\hline Additional views & $5-10 \mathrm{~min} / \mathrm{view}$ \\
\hline
\end{tabular}

- For melanoma, imaging of trunk lesions may include axillary and inguinal views; imaging of head and neck lesions may include anterior, posterior, and oblique views, and SPECT/CT may be deemed necessary.

\section{ADJUNCT IMAGING AND INTERVENTIONS}

The use of a $\gamma$ probe in the operating room may replace imaging in some institutions and can help to identify and pinpoint the region with the highest count rate for incision. Important note: The probe should be directed away from the activity at the injection site.

\section{PROCESSING}

To enhance low-count areas of the image, contrast enhancement should be used or the upper threshold of the computer display should be lowered.

\section{PRECAUTIONS}

- Leakage from the injection site may occur. Cover the injection site with gauze.

- If an intraoperative $\gamma$-probe is used to help locate the sentinel node, the tracer must be injected $15 \mathrm{~min}$ to $3 \mathrm{~h}$ before surgery. If surgery is delayed by 6 or more hours, obtaining another image before surgery to define further migration of the tracer to additional nodes is advised.

- Mapping should not be delayed more than $15 \mathrm{~h}$.

\section{REFERENCES}

1. Farrell MB, Mantel ES, Basso DA, et al. Lymphoscintigraphy: breast cancer. In: Quick-Reference Protocol Manual for Nuclear Medicine Technologists. Society of Nuclear Medicine and Molecular Imaging; 2014:218-220.

2. Farrell MB, Mantel ES, Basso DA, et al. Lymphoscintigraphy: melanoma. In: Quick-Reference Protocol Manual for Nuclear Medicine Technologists. Society of Nuclear Medicine and Molecular Imaging; 2014:221-223.

3. Waterstram-Rich K, Gilmore D. Inflammatory/tumor/oncology imaging and therapy. In: Nuclear Medicine and PET/CT Technology and Techniques, 8th ed. Mosby Elsevier; 2017:609-611.

4. Bluemel C, Herrmann K, Giammarile F, et al. EANM practice guidelines for lymphoscintigraphy and sentinel lymph node biopsy in melanoma. Eur J Nucl Med Mol Imaging. 2015;42:1750-1766.

5. Giammarile F, Alazrake N, Aarsvold JN, et al. The EANM and SNMMI practice guideline for lymphoscintigraphy and sentinel node localization in breast cancer. Eur J Nucl Med Mol Imaging. 2013;40:1932-1947. 